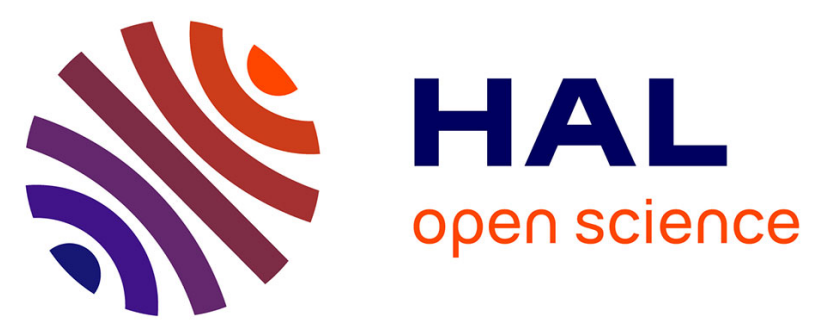

\title{
Learning to run the number line: the development of attentional shifts during single-digit arithmetic
}

Andrea Díaz-barriga Yáñez, Auriane Couderc, Léa Longo, Annabelle Merchie, Hanna Chesnokova, Emma Langlois, Catherine Thevenot, Jérôme Prado

\section{- To cite this version:}

Andrea Díaz-barriga Yáñez, Auriane Couderc, Léa Longo, Annabelle Merchie, Hanna Chesnokova, et al. Learning to run the number line: the development of attentional shifts during single-digit arithmetic. Annals of the New York Academy of Sciences, 2020, 1477 (1), pp.79-90. 10.1111/nyas.14464 . hal-02988660

\section{HAL Id: hal-02988660 \\ https://hal.science/hal-02988660}

Submitted on 10 Dec 2020

HAL is a multi-disciplinary open access archive for the deposit and dissemination of scientific research documents, whether they are published or not. The documents may come from teaching and research institutions in France or abroad, or from public or private research centers.
L'archive ouverte pluridisciplinaire HAL, est destinée au dépôt et à la diffusion de documents scientifiques de niveau recherche, publiés ou non, émanant des établissements d'enseignement et de recherche français ou étrangers, des laboratoires publics ou privés. 


\section{Learning to run the number line:}

\section{The development of attentional shifts during single-digit arithmetic}

Andrea Díaz-Barriga Yáñez ${ }^{1}$, Auriane Couderc ${ }^{1}$, Léa Longo ${ }^{1}$, Annabelle Merchie ${ }^{1}$, Hanna Chesnokova $^{1}$, Emma Langlois ${ }^{1}$, Catherine Thevenot ${ }^{2^{*}}$, \& Jérôme Prado ${ }^{1 *}$

${ }^{1}$ Lyon Neuroscience Research Center (CRNL), INSERM U1028 - CNRS UMR5292, University of Lyon, France

${ }^{2}$ Institut de Psychologie, Université de Lausanne, Switzerland

* These authors shared senior authorship

Corresponding Author:

Correspondence should be addressed to J.P. (jerome.prado@univ-lyon1.fr), Lyon Neuroscience Research Center, CH Le Vinatier, 95 bd Pinel, 69675 Bron Cedex, France, or C.T. (catherine.thevenot@ unil.ch), Institut de Psychologie, Université de Lausanne, Géopolis, CH-1015 Lausanne, Suisse

Short title: Learning to run the number line

Keywords: arithmetic; spatial attention; numerical cognition; development

Word count (excluding abstract, references, and figure legends): 5346 


\begin{abstract}
Recent studies suggest that solving single-digit subtraction and addition problems is associated with left and right shifts of attention in adults. Here, we explored the development of these spatial shifts in children from 7 to 11 . In two experiments, children were asked to solve single-digit addition, subtraction, and multiplication problems in which operands and arithmetic sign were shown sequentially. Although the first operand and the arithmetic sign were presented on the center of a screen, the second operand was presented either to the left or to the right visual field. We found that subtraction problems were increasingly associated with a leftward bias from 7 to 11 , such that problem solving was facilitated when the second operand was in the left visual field around age 10-11 (i.e., grade 5). We also found evidence that at least some children may already associate addition problems with the right side of space by age 9-10 (i.e., grade 4). No developmental increase in either leftward or rightward bias was observed for multiplication problems. We argue that these attentional shifts reflect the progressive automatization of calculation procedures that involve specific movements to the left or right of a sequential representation of numbers during subtraction and addition.
\end{abstract}




\section{Introduction}

Increasing evidence indicates that our ability to process numbers is grounded in spatial representations ${ }^{1}$. For example, small numbers are associated with the left side of space and large numbers with the right side of space. ${ }^{2-4}$ Numbers also automatically bias spatial attention, such that targets are detected faster in the left visual field (LVF) when they follow small numbers, and faster in the right visual field (RVF) when they follow large numbers. 5,6 Taken together, this suggests that numerical magnitudes may be represented on a mental number line (MNL) that is organized horizontally and in ascending order from left to right. ${ }^{3,7}$

Recent studies suggest that such spatial associations may not be limited to number processing per se, but might also be observed during arithmetic calculation in adults. For example, when adults are asked to estimate the result of a subtraction or an addition problem, they tend to underestimate the result of a subtraction and overestimate the result of an addition. ${ }^{8,9}$ Several explanations for this effect (termed operational momentum, OME) have been proposed. ${ }^{10-12}$ Nevertheless, one major explanation is that participants rely on attentional shifts along the MNL to estimate results of problems. In a recent study, we argued that such attentional shifts might occur during exact symbolic arithmetic as well. ${ }^{13}$ Specifically, we asked adults to solve single-digit arithmetic problems presented on a computer screen. While the first operand and the arithmetic sign were presented sequentially at the center of the screen, the second operand was presented either in the LVF or the RVF (see Fig. 1). Results indicated that participants were faster to solve addition problems when the second operand was presented in the RVF than the LVF, while they were faster to solve subtraction problems when the second operand was presented in the LVF than the RVF. Thus, spatial shifts of attention are elicited during exact single-digit arithmetic in adults. Importantly, no spatial bias was observed in multiplication problems (which are explicitly learned by rote in school). Thus, it is possible that these horizontal shifts of attention reflect calculation procedures relying on left-right movements along the MNL that have been automatized after years of practice with arithmetic calculation. ${ }^{14}$

To date, few studies have investigated the associations between arithmetic and space in children. However, available evidence suggests that these associations may be relatively late developing. For example, studies have failed to find an OME in 6- and 7-year-olds ${ }^{15}$ as well as in 9-year-olds. ${ }^{16}$ It has thus been suggested that associations between space and arithmetic might be the result of years of education and that an "unconscious shift of attention 
on the MNL becomes evident only with increasing expertise and automatization". ${ }^{16}$ This proposal is consistent with the idea that automatic leftward and rightward movements along a sequential representation of numbers might stem from the repeated use of deliberate counting during arithmetic learning in elementary school. ${ }^{14,17-19}$ Yet, to our knowledge, there is no evidence that increasing practice with arithmetic is associated with the emergence of shifts of attention along the MNL during mental calculation in children.

To test this hypothesis, we explored in two experiments the developmental emergence of spatial biases during mental calculation in children from 7 to 11 . Using the same paradigm as Mathieu and colleagues ${ }^{13}$, one group of children was presented with single-digit addition and subtraction problems (Experiment 1) and another group with single-digit addition and multiplication problems (Experiment 2). We expected addition problems to be increasingly associated with a rightward bias with age. Conversely, we expected subtraction problems to be increasingly associated with a leftward bias with age. However, there should be no emergence of associations between multiplication problems and space, as these problems are directly retrieved from memory and may not involve calculation procedures relying on shifts along the MNL.

\section{Experiment 1}

\section{Materials and Methods}

\section{Participants}

Data collection was conducted in two different time waves. The first wave of data collection (Wave 1) occurred between December 2014 and March 2015, and the second wave of data collection (Wave 2) occured between November and December 2019. Participants in Wave 1 were recruited from three private elementary schools in the area of Lyon in France. They consisted in 63 children aged between 7 and 11 years. Data from 6 children were excluded from the analysis for medical (dyscalculia, deafness) and behavioral (lack of answers for one of the two types of operations) reasons. Thus, data from Wave 1 came from the remaining 57 children (32 Female, Mean age $=9.44$ years, $S D=.81$ ). Participants in Wave 2 were recruited from one private primary school in the area of Lyon $(\mathrm{N}=29)$ and via a social 
media website $(\mathrm{N}=18)$. Thus, 47 children aged between 7 and 10 years were in Wave 2 (20 Female, Mean age=8.75 years, $\mathrm{SD}=.61$ ). Data from three children were further removed because they were outliers (see below). As such, final data came from 101 children (50 female) with ages between 7 and 11 years (Mean age=9.10, SD=.80). All children were native French speakers. Overall, 47 children were in 3rd grade, 33 children were in 4th grade, and 21 children were in 5th grade. The experiment was performed in accordance with the ethical standards established by the Declaration of Helsinki.

\section{Procedure}

All children were tested in one single session that lasted approximately 30 minutes. Children from Wave 1 were tested in a quiet area of their school, whereas children from Wave 2 were tested either in the lab or in a quiet area of their school. Each child was tested individually and gave verbal assent to participant. Parent's written consents were also obtained. The testing session started with the Math Fluency test from the Woodcock-Johnson battery ${ }^{20}$ to assess children's arithmetic skills (see below), followed by the experimental task. 13

\section{Measures}

Math fluency: Children's arithmetic skills were first assessed with the WoodcockJohnson III Math Fluency subtest. The Math Fluency is a paper-and-pencil test that includes single-digit addition, subtraction, and multiplication problems. Children needed to solve as many problems as possible within 3 minutes. ${ }^{20}$

Experimental task: The experimental task was computer-based and adapted from Mathieu and colleagues. ${ }^{13}$ Small arithmetic problems included pairs of non-identical operands between 1 and 5 [(2, 1); $(3,1) ;(3,2) ;(4,1) ;(4,2) ;(4,3) ;(5,1) ;(5,2) ;(5,3) ;(5$, 4)], and large arithmetic problems included pairs of non-identical operands between 5 and 9 $[(6,5) ;(7,5) ;(7,6) ;(8,5) ;(8,6) ;(8,7) ;(9,5) ;(9,6) ;(9,7) ;(9,8)]$. Both small and large problems contained the number 5 in order to have the same number of problems in both categories. These pairs of non-identical operands were used to construct 20 addition problems (10 small problems and 10 large problems) and 20 subtraction problems (10 small problems and 10 large problems) with their second operand presented once to the right and once to the left, resulting in a total of 80 problems (i.e., 40 addition problems and 40 subtraction 
problems). For both addition and subtraction problems, the larger of the two operands was always presented first. This ensured that results from the subtraction problems were positive and that children could not anticipate the type of problem to come. The presentation of the trials was pseudo-randomized, so that no more than three problems of the same type (i.e., same size and spatial location of the second operand) would appear consecutively. Four scenarios were created by generating 4 random lists of trials. Each scenario was separated into 2 runs of 40 operations. The experiment systematically started with a practice run of 8 trials including tie problems (e.g. 7 - 7), problems with 0 (e.g. $3+0)$, and problems with small and large operands (e.g. $7+3)$.

In each trial, operands and arithmetic sign were displayed in white Times New Roman 36-point font on a black background (see Fig. 1). Each trial started with the presentation of a white fixation dot for $500 \mathrm{~ms}$ followed by the presentation of the first operand that lasted an additional $500 \mathrm{~ms}$ on screen. After a first delay of $500 \mathrm{~ms}$, either a + or a - sign appeared on screen for $150 \mathrm{~ms}$ at the center of the screen. A second delay of $300 \mathrm{~ms}$ separated the disappearance of the arithmetic sign from the second operand. This delay was chosen because it was the delay for which arithmetic shifts were maximal in Mathieu et al., 2016. The second operand was then displayed for $150 \mathrm{~ms}$, either $5^{\circ}$ to the left or $5^{\circ}$ to the right of the center of the screen. Children needed to solve the calculation verbally in less than 5 seconds, otherwise the software automatically moved on to the next arithmetic problem.

All response times (RTs) were recorded through a headset microphone and corresponded to the period between the presentation of the second operand and the onset of the answer. The experiment was controlled by the DmDX software ${ }^{21}$ and RTs were checked off-line and manually adjusted with CheckVocal ${ }^{22}$ for each participant. Before the experiment started, children were given the instructions printed in a A4 white sheet and were able to ask any questions if needed. Children were located $44 \mathrm{~cm}$ from the 15 -inch computer screen. A chin-rest was used to avoid head movements.

\section{Results and discussion}

Only correct responses were analyzed. Correct trials constituted $90.33 \%$ of trials for small problems, but only $62.78 \%$ of trials for large problems. To maximize power and ensure that our results would not be confounded by differences in accuracy between older and younger children, we exclusively focused our analyses on small problems. Small addition 
problems $(91.5 \%)$ were responded more accurately than small subtraction problems $(89.15 \%)$ $(t(103)=2.09, p=.04)$. For each participant and operation, we subtracted the mean RT of trials in which the second operand appeared on the right from the mean RT of trials in which the second operand appeared on the left. This difference in RT (dRT) served as dependent variable in the following analyses (see Ref. 13). Outliers (dRT smaller or greater than 2.5 SDs from the mean for each participant in either small addition or subtraction problems) were removed from the analyses (this corresponded to 3 children).

\section{Descriptive statistics}

Descriptive statistics for age, Math fluency scores, dRTs for small addition and subtraction problems, as well as RTs for small addition and subtraction problems are shown in Table 1.

Preliminary analysis

Independent $t$ tests were conducted to test whether there was an effect of Wave (Wave 1 vs Wave 2) and gender on dRTs for small addition and subtraction problems. Results revealed no Wave $(t(99)=1.09, p=.28$ for $\mathrm{dRT}$ in small addition problems, and $t(99)=.08$, $p=.94$ for small subtraction problems $)$ or gender $(t(99)=.46, p=.64$ for dRT for small addition problems, and $t(99)=.67, p=.50$ for small subtraction problems ${ }^{1}$ ) effects in any of the dRTs.

dRTs analyses

As children get older (and calculation procedures would become more automatized), we predicted that dRTs would become more positive for addition problems (i.e., an increase in rightward bias) and more negative for subtraction problems (i.e., an increase in leftward bias). Thus, dRTs for addition and subtraction problems were analyzed in multiple regression analyses with age as the main predictor. Because age was positively correlated with math fluency scores ( $r=.30, p<.01$, two-tailed) and negatively correlated with overall RTs for small addition problems $(r=-.22, p<.05$, two-tailed) and small subtraction problems $(r=-.20, p<.05$, two-tailed), these predictors were also included in the multiple regression analyses in order to investigate the amount of unique contributions of age to differences in dRTs (see Table 2).

\footnotetext{
${ }^{1}$ Levene's test was significant, suggesting a violation of the equal variance assumption, thus a Mann-Whitney test was conducted. Results also revealed no gender effect, $\mathrm{U}=1238, p=.32, z=-.25$ for small addition problems, and $\mathrm{U}=1190, p=.98, z=-.58$ for small subtraction problems.
} 
For both multiple regression analyses, the Durbin-Watson test was found to be within acceptable parameters (2.34 and 2.13 respectively), suggesting that the assumption of independent errors was met and that results may apply to a wider population. ${ }^{23}$ Multicollinearity was also systematically checked using the variance inflation factor (VIF). The largest VIF was well below 10 for both regressions. Similarly, the tolerance data were all within acceptable boundaries (all greater than 0.1). Therefore, it was concluded that there was no collinearity within the data. ${ }^{23}$

Over and above differences in math fluency and overall RTs, dRTs for small addition problems did not increase with age $\left(t(97)=-.17, p=.57\right.$, one-tailed ${ }^{2}$ ) (see Table 2 and Fig. 2A). Such a lack of positive relationship between age and dRTs for small addition problems is not consistent with our hypotheses, as we predicted an increase in rightward bias for addition problems while children get older and therefore should automatize addition procedures. One possibility is that the bias might already be present in the youngest children investigated (such that the rightward bias might develop earlier than $3^{\text {rd }}$ grade). To investigate this possibility, we tested whether dRTs for small addition problems were larger than 0 in children from $3^{\text {rd }}$ grade (i.e., approximately age $8-9$ ), $4^{\text {th }}$ grade (i.e., approximately age 9-10), and $5^{\text {th }}$ grade (i.e., approximately age 10-11) in our sample. One-sample t-tests indicated that dRTs for small addition problems were not larger than 0 in children from $3^{\text {rd }}(t(46)=.92$, $p=.18$, one-tailed, $d=.13), 4^{\text {th }}(t(32)=-1.46, p=.92$, one-tailed, $d=-.25)$, or $5^{\text {th }}$ grade $(t(20)$ $=.14, p=.45$, one-tailed, $d=.03$ ). Therefore, contrary to our hypothesis, we did not find any evidence for either an increase or an early presence of a rightward bias in small addition problems in this sample of elementary school children. Although it is always difficult to interpret a null result, potential reasons for this lack of effect will be discussed in the general discussion.

In line with our hypotheses, however, we found a significant negative relationship between age and dRTs for small subtraction problems $\left(t(97)=-1.89, p=0.03\right.$, one-tailed ${ }^{3}$, see Table 2). In other words, dRTs for small subtraction problems were more negative in older than younger children (see Fig. 2B). To further explore this effect, we tested whether dRTs

\footnotetext{
${ }^{2}$ Our hypothesis was unidirectional because we only expected dRTs for small addition problems to increase (and not decrease) with age.

${ }^{3}$ Our hypothesis was unidirectional because we only expected dRTs for small subtraction problems to decrease (and not increase) with age.
} 
for small subtraction problems were smaller than 0 in children from grades 3, 4, and 5 in our sample. We found that dRTs for small subtraction problems were not smaller than 0 in $3^{\text {rd }}(t$ (46) $=1.93, p=.97$, one-tailed, $d=.28)$ and $4^{\text {th }}$ grade $(t(32)=.70, p=.76$, one-tailed, $d=.12)$. However, dRTs for small subtraction problems were significantly smaller than 0 in $5^{\text {th }}$ grade $(t(20)=-2.36, p=.01$, one-tailed, $d=-.51)$. Therefore, our results provide evidence for an increase in leftward bias when children solve subtraction problems over the course of elementary school, such that children exhibit shorter response times when the second operand is on the LVF (as compared to the RVF) around the ages of 10-11 (i.e., $5^{\text {th }}$ grade). This finding is in line with the proposal that, as children get older and practice arithmetic problem solving, they may automatize calculation procedures relying on attentional shifts along the MNL. ${ }^{13}$ In the case of subtraction, this would involve shifting attention to the left of the MNL, such that the " - " sign may automatically direct attention to the left side of space during problem solving.

These shifts of attention, however, should not be observed if answers are not calculated but directly retrieved from memory. ${ }^{14}$ For example, Mathieu and colleagues ${ }^{13}$ found that the position of the second operand (LVF versus RVF) did not affect solving times during multiplication problem solving. This is consistent with the idea that solving multiplication problems does not involve calculation procedures but instead direct retrieval from memory (because single-digit multiplication problems are learned by rote in school; ${ }^{13}$ ). Thus, in Experiment 2, we presented another group of children with multiplication (as well as addition) problems using the same paradigm as in Experiment 1.

\section{Experiment 2}

\section{Materials and Methods}

\section{Participants}

As for Experiment 1, data collection was conducted in two different time waves. Participants in Wave 1 were recruited from one private primary school in the area of Lyon, France. They consisted in 38 children aged between 8 and 11 years ( 21 Female, Mean age $=10$ years, $\mathrm{SD}=.65$ ). Participants in Wave 2 were recruited from another private elementary 
school in the same area $(\mathrm{N}=22)$ and via a social media website $(\mathrm{N}=9)$. Thus, 31 children aged between 8 and 10 years were in Wave 2 (20 Female, Mean age=9.42 years, SD=.48). Data from one child were further removed because they were outliers (see below). As such, the overall sample came from 68 children (41 female) with ages between 8 and 11 years (Mean age $=9.74$ years, $\mathrm{SD}=.65$ ). All children were native French speakers. Overall, 42 children were in $4^{\text {th }}$ grade and 26 children were in $5^{\text {th }}$ grade. The experiment was performed in accordance with the ethical standards established by the Declaration of Helsinki.

\section{Procedure}

Experiment 2 followed the same procedure as Experiment 1, with all children being tested in one single session. However, the testing session lasted between 30 and 40 minutes because the experimental task included two more runs (see below). The testing session started with the Math Fluency test from the Woodcock-Johnson battery ${ }^{20}$ followed by the experimental task.

\section{Measures}

The Woodcock-Johnson Math Fluency subtest used in Experiment 2 was the same as in Experiment 1. Regarding the experimental task, the pairs of non-identical operands, the classification of problems (i.e. small and large), the apparatus, and the stimulus timing were the same as in Experiment 1. Hence, only full details for the stimuli and for the experimental procedure of Experiment 2 are provided next.

For Experiment 2, the pairs of non-identical operands were used to construct 20 addition problems (10 small problems and 10 large problems) and 20 multiplication problems (10 small problems and 10 large problems) with their second operand presented once to the right and once to the left, resulting in 40 addition problems and 40 multiplication problems. The largest operand could be presented as either the first or the second operand, such that each problem was presented once for each order of presentation of operand (first vs second position). Therefore, there were a total of 160 problems, which were separated into 4 runs of 40 operations. The trials were pseudo-randomized and 4 different scenarios based on 4 different lists were created (with 4 runs in each scenario).

\section{Results and discussion}


As in Experiment 1, only correct responses in small arithmetic problems were analyzed. Correct trials constituted $89.4 \%$ of trials for small problems, but only $56.89 \%$ of trials for large problems. There was no accuracy difference between small addition problems $(89.75 \%)$ and small multiplication problems $(89.05 \%)(t(68)=.68, p=.50)$. dRTs were also calculated and served as dependent variable in the following analyses. Outliers (dRT smaller or greater than 2.5 SDs from the mean for each participant in either small addition or multiplication problems) were removed from the analyses (this corresponded to 1 child). Additionally, data from one child on the WJ Math fluency task was considered as missing in further statistical analyses due to failure to understand task instructions. Analyses were therefore conducted using all available data using pairwise deletion to avoid bias and reduction in power.

\section{Descriptive statistics}

Descriptive statistics for age, Math fluency scores, dRTs for small addition and multiplication problems, as well as overall RTs for all small addition and multiplication problems are shown in Table 3.

\section{Preliminary analysis}

Independent $t$ tests were conducted to test whether there was an effect of Wave (Wave 1 vs Wave 2) and gender on dRTs for small addition and multiplication problems. Results revealed no Wave $(t(66)=1.7, p=.10$ for $\mathrm{dRT}$ in small addition problems, and $t(66)=-.87$, $p=.39$ for small multiplication problems) or gender $(t(66)=-1.39, p=.17$ for $\mathrm{dRTs}$ in small addition problems, and $t(66)=-1.35, p=.18$ for small multiplication problems) effects in any of the dRTs.

\section{dRTs analyses}

dRTs for addition and multiplication problems were analyzed in multiple regression analyses with age as the main predictor of interest. Just as in Experiment 1, we expected addition problems to be increasingly associated with a rightward bias in older than younger children. We also predicted a lack of developmental increase in either leftward or rightward bias for multiplication problems. As stated before, these problems are thought to involve direct retrieval from memory and may not involve calculation procedures relying on movements along the MNL. As in Experiment 1, math fluency scores and overall RTs for 
either small addition or small multiplication problems were included in the multiple regression analyses. Also like in Experiment 1, for both multiple regression analyses, the Durbin-Watson test was systematically checked and was found to be within acceptable parameters (1.85 and 2 respectively), suggesting that the assumption of independent errors was met and that results may apply to a wider population. ${ }^{23}$ Multicollinearity was also checked using the VIF. The largest VIF was well below 10 for both regressions. Similarly, the tolerance data were all within acceptable boundaries (all greater than 0.1). Therefore, it was concluded that there was no collinearity within the data. ${ }^{23}$

Results showed that, over and above differences in math fluency and overall RTs, dRTs for small addition problems increased with age $\left(t(63)=1.67, p=0.0499\right.$, one-tailed ${ }^{4}$, see Table 4 and Fig. 3A). In other words, dRTs for small addition problems were more positive for older than younger children. Further analyses showed that dRTs for small addition problems were larger than 0 in children from both $4^{\text {th }}(t(41)=2.96, p=.003$, one-tailed, $d=.46)$ and $5^{\text {th }}$ grade $(t(25)=3.35, p=.001$, one-tailed, $d=.66)$. Thus, in this sample, children already exhibited shorter response times when the second operand was on the RVF (as compared to the LVF) in $4^{\text {th }}$ grade. Furthermore, this rightward bias increased with age. This finding suggests that, much like subtraction problem solving (Experiment 1), addition problem solving may be characterized by an increase of attentional shifts along the MNL as children get older. In the case of addition, however, these shifts might involve moving to the right of the MNL, such that the " + " sign may automatically direct attention to the right side of space during problem solving. Note, however, that these results are inconsistent with our own findings in Experiment 1, since neither a rightward bias nor a developmental increase in rightward bias was found in addition problems in Experiment 1. Potential reasons for the inconsistencies between experiments are discussed in the general discussion.

In contrast to small addition problems, we did not find any relationship between age and dRTs for multiplication problems $(t(63)=.29, p=.77$, two-tailed) (see Table 4 and Fig. 3B). Follow up analyses also showed that dRTs in small multiplication problems were not different than 0 in either $4^{\text {th }}(t(41)=-.84, p=.41$, two-tailed $)$ or $5^{\text {th }}$ grade $(t(25)=.16, p=.89$, two-tailed). Thus, we found no evidence of a leftward or rightward bias during multiplication problem solving in elementary school children. This is consistent with the fact that answers of multiplication problems are mainly learned by rote in school and therefore never really

\footnotetext{
${ }^{4}$ Our hypothesis was unidirectional because we only expected dRTs for small addition problems to increase (and not decrease) with age.
} 
calculated by children. The lack of spatial bias with multiplication problems also makes it unlikely that the rightward bias observed with addition problems (in Experiment 2) is due to the size of the answer. Indeed, addition outcomes are always larger than both of the operands involved. Thus, it is possible that the rightward bias observed may simply result from an association between relatively large numbers and the right space of the MNL (e.g. ${ }^{2-4,24}$ ), rather than a calculation procedure per se. However, outcomes of multiplication problems in Experiment 2 were larger, or as large as, the operands involved (because there were no multiplications involving 0). Thus, the lack of rightward bias with multiplication problems discards the hypothesis that the rightward bias observed with addition problems is due to the size of the answer.

\section{General Discussion}

Several studies suggest that arithmetic calculation is associated with attentional shifts in adults. ${ }^{13,25-28}$ To our knowledge, however, no study has investigated how and when these shifts emerge in children. The aim of the two experiments presented in this paper was to investigate the developmental emergence of spatial biases during mental calculation in children from 7 to 11. Children were asked to verbally solve single-digit addition, subtraction, and multiplication problems. Operands and arithmetic signs were presented sequentially. Although the first operand and the arithmetic sign were presented on the center of the screen, the second operand was displayed either in the left or in the right side of the fixation.

In Experiment 1, we found that small subtraction problems were increasingly associated with a leftward bias from 7 to 11 , such that only children at the end of elementary school (i.e., grade 5 or age 10-11) showed an association between subtraction problems and the left side of space. We also found in Experiment 2 that at least some children may show an association between small addition problems and the right side of space as early as grade 4 (i.e., age 9-10), and that this rightward bias tends to increase at least until the end of elementary school. We can see at least 3 potential explanations for the emergence of these associations.

First, because operands were kept constant across operations, results were overall smaller for subtraction than for addition problems. It could then be argued that number-space associations might have contributed to the difference in spatial bias between subtraction and 
addition. However, this is unlikely because number-space associations have been demonstrated before 3rd grade in previous studies ${ }^{29,30}$ and the association between subtraction and the left side of space was only observed in 5th grade in Experiment 1. Furthermore, no spatial association was observed for multiplication problems in Experiment 2, despite the fact that multiplication problems lead to results that are even higher than addition problems.

Second, it is possible that with practice children progressively associate the "_" and the "+" signs with simple heuristics such as "the result of a subtraction should always be smaller than the first operand" and "the result of an addition should always be larger than the first operand". ${ }^{11,24,31}$ Simple mappings between arithmetic signs and space could then explain an operation-dependent spatial bias. This account, however, would also predict an association between the multiplication sign and the right side of space given that results of multiplication problems are larger than the first operand (at least when it is greater than 1). Yet, there was no such effect in Experiment 2.

Third, a number of recent studies have suggested that the repeated use of counting when young children solve basic addition and subtraction problems might lead to an automatization of these counting procedures, rather than to the construction of a network of arithmetic facts in memory as posited by prior literature. ${ }^{14,17-19}$ For example, studies in adults ${ }^{17,19}$ and 10 -year-old children ${ }^{18}$ show that the time participants take to solve very small addition problems is not constant but increases linearly as a function of the distance between the original operand and the sum. This suggests that, even if they might not be aware of it, adults and skilled children might solve these basic problems by rapidly "moving" from a source to a target number along the MNL. Given the left-to-right orientation of that MNL in children and adults ${ }^{1,29,32}$, these forward and backward movements are likely to resemble rightward and leftward shifts of attention. Because the automaticity of these shifts during calculation is likely to rely on children's practice with counting and attentional skills (both of which arguably increase from 7 to 11 ), such an account may explain our findings. That is, spatial shifts of attention may emerge in elementary school because children automatize movements along the MNL that are specific to subtraction and addition.

It is important to note, however, that our results regarding addition problems are inconsistent. That is, we observed a rightward bias in Experiment 2 but not in Experiment 1. There are a number of possible explanations for this. However, two (non-exclusive) 
speculations may come to mind. First, it is possible that, in contrast to children in Experiment 2, a majority of children from Experiment 1 may have retrieved answers of small addition problems from long-term memory. For instance, some children might have learned answers of some addition problems by rote, as the French math curriculum emphasizes that small addition problems can be memorized in tables ${ }^{33}$. This would make small addition problems somewhat similar to multiplication problems and might explain a lack of spatial association. Note that this explanation remains consistent with the observation that the same children show a leftward bias with subtraction because subtraction problems are seldom learned by rote in the French curriculum. ${ }^{33}$ Unfortunately, this explanation is speculative because we did not collect information on the way children were taught addition problems in our study. However, variability in the methods used to teach addition between schools and classrooms may account from variability in our results.

A second possibility is that automatizing calculation procedures relying on movements along the MNL is likely to require a great deal of practice with counting and arithmetic calculation. Because this may also critically differ between children from different schools and classrooms, it is possible that children from Experiment 1 may have been exposed to less extensive practice with arithmetic calculation than children from Experiment 2. An examination of arithmetic performance between children from Experiment 1 and 2 suggests that it might be the case. For example, 4th- and 5th-graders in Experiment 2 had a significantly higher score on the Math fluency test than 4th- and 5th-graders in Experiment 1 $(t(119)=2.16, p=.017$, one-tailed $)$. This raises the possibility that we might have failed to observe a rightward bias in children from Experiment 1 because these children were not as fluent with addition problem solving as children from Experiment 2. Note, however, that this possibility is mitigated by the fact that Math fluency scores were not significantly related to dRTs for small addition problems in either Experiment 1 or Experiment 2. Therefore, children from Experiment 1 and Experiment 2 might also differ with respect to cognitive skills that have been shown to affect the automaticity of counting procedures, such as working memory and processing speed. ${ }^{14,17-19}$ Because these skills were not measured here, future studies are needed to better understand individual variability in the development of attentional shifts during addition problem solving.

Nonetheless, our findings provide the first evidence for the emergence of associations between arithmetic processing and space in elementary school children. Specifically, we 
provide evidence that at least some children already associate addition problems with the right side of space by age 9-10 (i.e., grade 4), whereas subtraction problems are associated with the left side of space by age 10-11 (i.e., grade 5). However, these spatial associations may also depend on the level of arithmetic fluency in children, as well as on the way arithmetic is taught in school. Future studies are needed to investigate if these associations become even more salient in older children, and how they relate to mathematical skills. 


\section{Acknowledgments}

The concept and design of the present research project was developed by Catherine Thevenot and Jérôme Prado. Data collection for Wave 1 was conducted by Auriane Couderc. Data collection for Wave 2 was conducted by Andrea Díaz-Barriga Yáñez, Léa Longo, Hanna Chesnokova, and Emma Langlois. Interpretation of the data, writing, and editing of the manuscript were conducted by Andrea Díaz-Barriga Yáñez, Catherine Thevenot, and Jérôme Prado. Statistical analyses were conducted by Andrea Díaz-Barriga Yáñez, Annabelle Merchie, and Jérôme Prado. Andrea Díaz -Barriga Yáñez takes responsibility for the integrity of the data analyzed. We would like to thank all of the schools and the children who took part in the experiments.

\section{Competing interests}

None 


\section{References}

1. Fischer MH, Shaki S. Spatial biases in mental arithmetic. $Q J$ Exp Psychol. 2014;67(8):1457-1460. doi:10.1080/17470218.2014.927516

2. Dehaene S. The number sense. How the mind creates mathematics.; 1997. doi: $10.2307 / 2589308$

3. Dehaene S, Bossini S, Giraux P. The Mental Representation of Parity and Number Magnitude. J Exp Psychol Gen. 1993;122(3):371-396. doi:10.1037/00963445.122.3.371

4. Wood, G., Willmes, K., Nuerk, H.-C., \& Fischer MH. On the cognitive link between space and number: a meta-analysis of the SNARC effect. Psychol Sci $Q$. 2008;50(4):489-525.

5. Dodd MD, Van der Stigchel S, Adil Leghari M, Fung G, Kingstone A. Attentional SNARC: There's something special about numbers (let us count the ways). Cognition. 2008;108(3):810-818. doi:10.1016/j.cognition.2008.04.006

6. Fischer MH, Castel AD, Dodd MD, Pratt J. Perceiving numbers causes spatial shifts of attention. Nat Neurosci. 2003;6(6):555-556. doi:10.1038/nn1066

7. Gevers W, Reynvoet B, Fias W. The mental representation of ordinal sequences is spatially organized Wim. Cognition. 2003;87:B87-B95. doi:10.1016/S0

8. Knops A, Viarouge A, Dehaene S. Dynamic representations underlying symbolic and nonsymbolic calculation: Evidence from the operational momentum effect. Attention, Perception, Psychophys. 2009;71(4):803-821. doi:doi:10.3758/APP.71.4.803

9. McCrink K, Dehaene S, Dehaene-Lambertz G. Moving along the number line: Operational momentum in nonsymbolic arithmetic. Percept Psychophys. 2007;69(8):1324-1333. doi:10.3758/BF03192949 
10. Chen Q, Verguts T. Spatial intuition in elementary arithmetic: A neurocomputational account. PLoS One. 2012;7(2):1-8. doi:10.1371/journal.pone.0031180

11. McCrink K, Wynn K. Operational momentum in large-number addition and subtraction by 9-month-olds. J Exp Child Psychol. 2009;103(4):400-408. doi:10.1016/j.jecp.2009.01.013

12. Pinhas M, Fischer MH. Mental movements without magnitude? A study of spatial biases in symbolic arithmetic. Cognition. 2008;109(3):408-415. doi:10.1016/j.cognition.2008.09.003

13. Mathieu R, Gourjon A, Couderc A, Thevenot C, Prado J. Running the number line: Rapid shifts of attention in single-digit arithmetic. Cognition. 2016;146:229-239. doi:10.1016/j.cognition.2015.10.002

14. Fayol M, Thevenot $\mathrm{C}$. The use of procedural knowledge in simple addition and subtraction problems. Cognition. 2012;123(3):392-403. doi:10.1016/j.cognition.2012.02.008

15. Knops A, Zitzmann S, McCrink K. Examining the presence and determinants of operational momentum in childhood. Front Psychol. 2013;4(JUN):1-14. doi:10.3389/fpsyg.2013.00325

16. Kucian K, Plangger F, O’Gorman R, Von Aster M. Operational momentum effect in children with and without developmental dyscalculia. Front Psychol. 2013;4(NOV):13. doi:10.3389/fpsyg.2013.00847

17. Barrouillet $\mathrm{P}$, Thevenot $\mathrm{C}$. On the problem-size effect in small additions: Can we really discard any counting-based account? Cognition. 2013;128(1):35-44. doi:10.1016/j.cognition.2013.02.018

18. Thevenot C, Barrouillet P, Castel C, Uittenhove K. Ten-year-old children strategies in 
mental addition: A counting model account. Cognition. 2016;146:48-57.

doi:10.1016/j.cognition.2015.09.003

19. Uittenhove K, Thevenot C, Barrouillet P. Fast automated counting procedures in addition problem solving: When are they used and why are they mistaken for retrieval? Cognition. 2016;146:289-303. doi:10.1016/j.cognition.2015.10.008

20. Woodcock, R. W., McGrew, K. S., \& Mather N. Woodcock-Johnson III Tests of cognitive abilities. Itasca, IL: Riverside Publishing; 2001.

21. Forster K, Forster J. DMDX: A windows display program with millisecond accuracy. Behav Res methods, instruments, Comput. 2003;35(1):116-124. http://dx.doi.org/10.3758/BF03195503.

22. Protopapas A. CheckVocal: A program to facilitate checking the accuracy and response time of vocal responses from DMDX. Behav Res Methods. 2007;39(4):859862. doi:10.3758/BF03192979

23. Field A. Discovering Statistics Using SPSS. London: SAGE Publications Ltd; 2009. doi:10.1017/CBO9781107415324.004

24. Hartmann M, Mast FW, Fischer MH. Spatial biases during mental arithmetic: Evidence from eye movements on a blank screen. Front Psychol. 2015;6(JAN):1-8. doi:10.3389/fpsyg.2015.00012

25. Liu Di, Cai D, Verguts T, Chen Q. The Time Course of Spatial Attention Shifts in Elementary Arithmetic. Sci Rep. 2017;7(1):1-8. doi:10.1038/s41598-017-01037-3

26. Pinheiro-Chagas P, Dotan D, Piazza M, Dehaene S. Finger Tracking Reveals the Covert Stages of Mental Arithmetic. Open Mind Discov Cogn Sci. 2017;1(1):30-41. doi:10.1162/opmi

27. Zhu R, Luo Y, You X, Wang Z. Spatial Bias Induced by Simple Addition and 
Subtraction: From Eye Movement Evidence. Perception. 2018;47(2):143-157. doi:10.1177/0301006617738718

28. Zhu R, You X, Gan S, Wang J. Spatial Attention Shifts in Addition and Subtraction Arithmetic: Evidence of Eye Movement. Perception. 2019;48(9):835-849. doi:10.1177/0301006619865156

29. Hoffmann D, Hornung C, Martin R, Schiltz C. Developing number-space associations: SNARC effects using a color discrimination task in 5-year-olds. J Exp Child Psychol. 2013;116(4):775-791. doi:10.1016/j.jecp.2013.07.013

30. Yang T, Chen C, Zhou X, Xu J, Dong Q, Chen C. Development of spatial representation of numbers: A study of the SNARC effect in Chinese children. $J$ Exp Child Psychol. 2014;117(1):1-11. doi:10.1016/j.jecp.2013.08.011

31. Marghetis T, Núñez R, Bergen BK. Doing arithmetic by hand: Hand movements during exact arithmetic reveal systematic, dynamic spatial processing. $Q J \operatorname{Exp}$ Psychol. 2014;67(8):1579-1596. doi:10.1080/17470218.2014.897359

32. Opfer JE, Thompson CA, Furlong EE. Early development of spatial-numeric associations: evidence from spatial and quantitative performance of preschoolers. Dev Sci. 2010;13(5):761-771. doi:10.1111/j.1467-7687.2009.00934.x

33. Blanquer J-M. Enseignement du calcul : un enjeu majeur pour la maîtrise des principaux éléments de mathématiques à l'école primaire.; 2018. https://www.education.gouv.fr/pid285/bulletin_officiel.html?cid_bo=128731. 


\section{Figures}

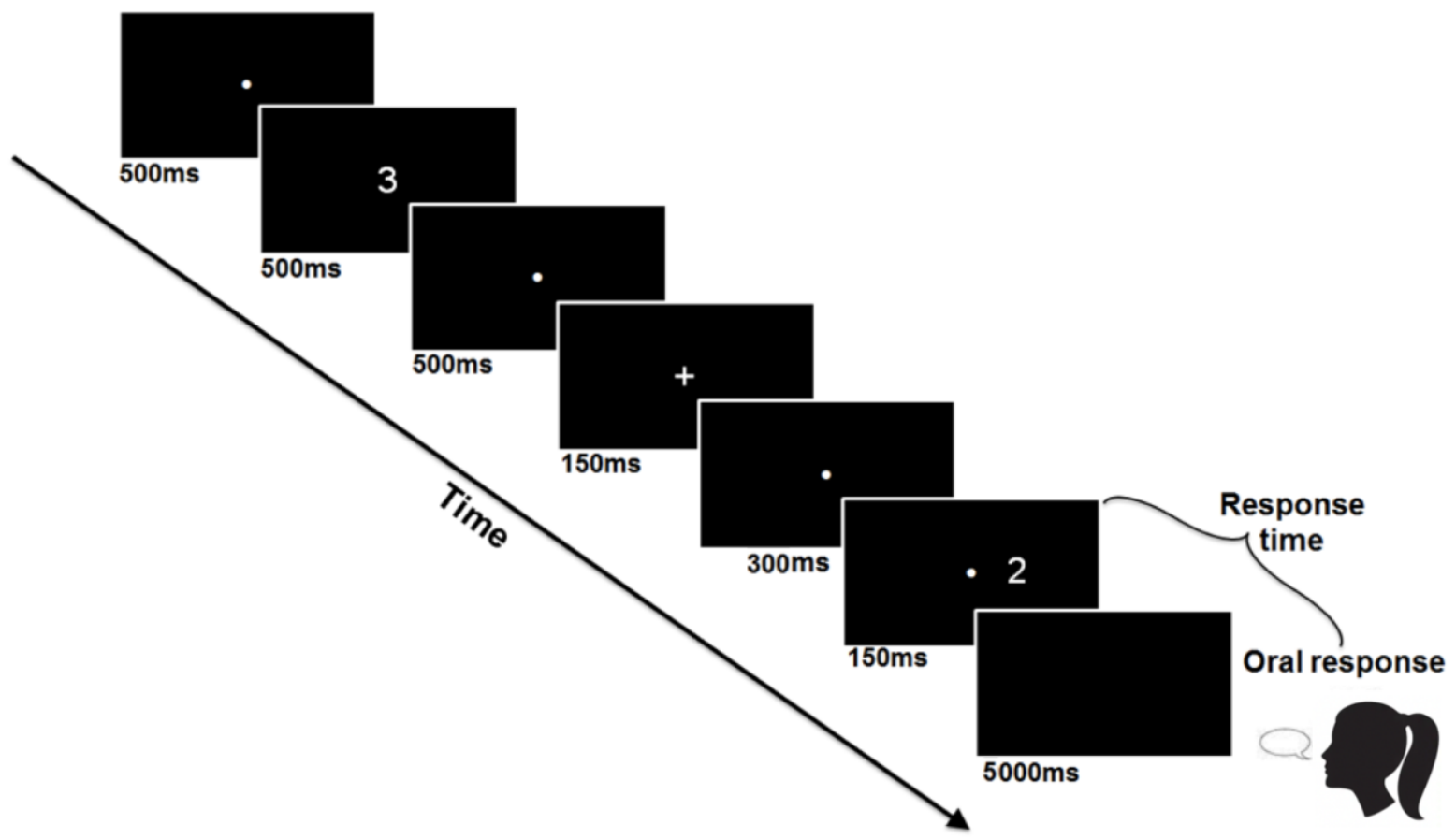

Figure 1. Sequence and timing of a sample trial. 

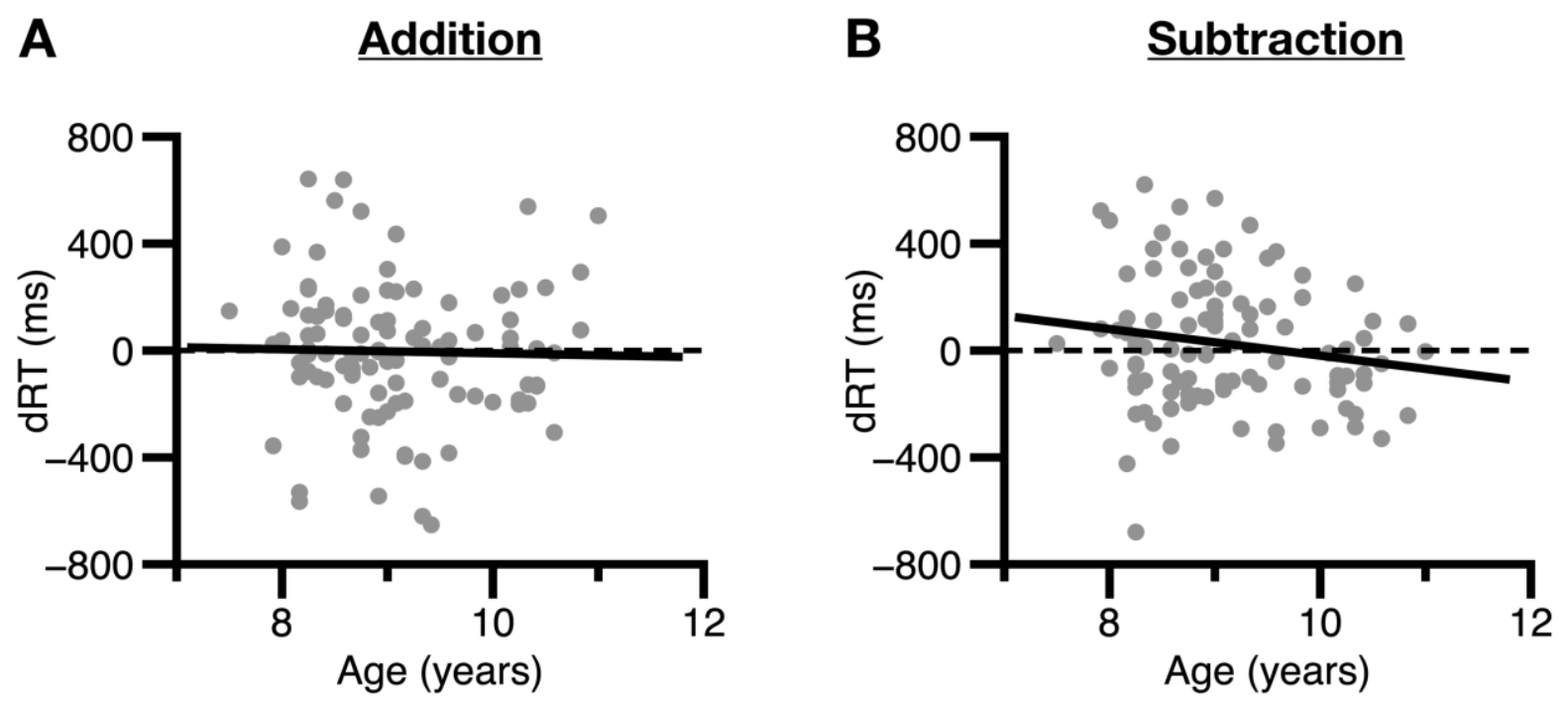

Figure 2. Relationship between age and dRTs for small arithmetic problems in Experiment 1. (A) Small addition problems. (B) Small subtraction problems. 

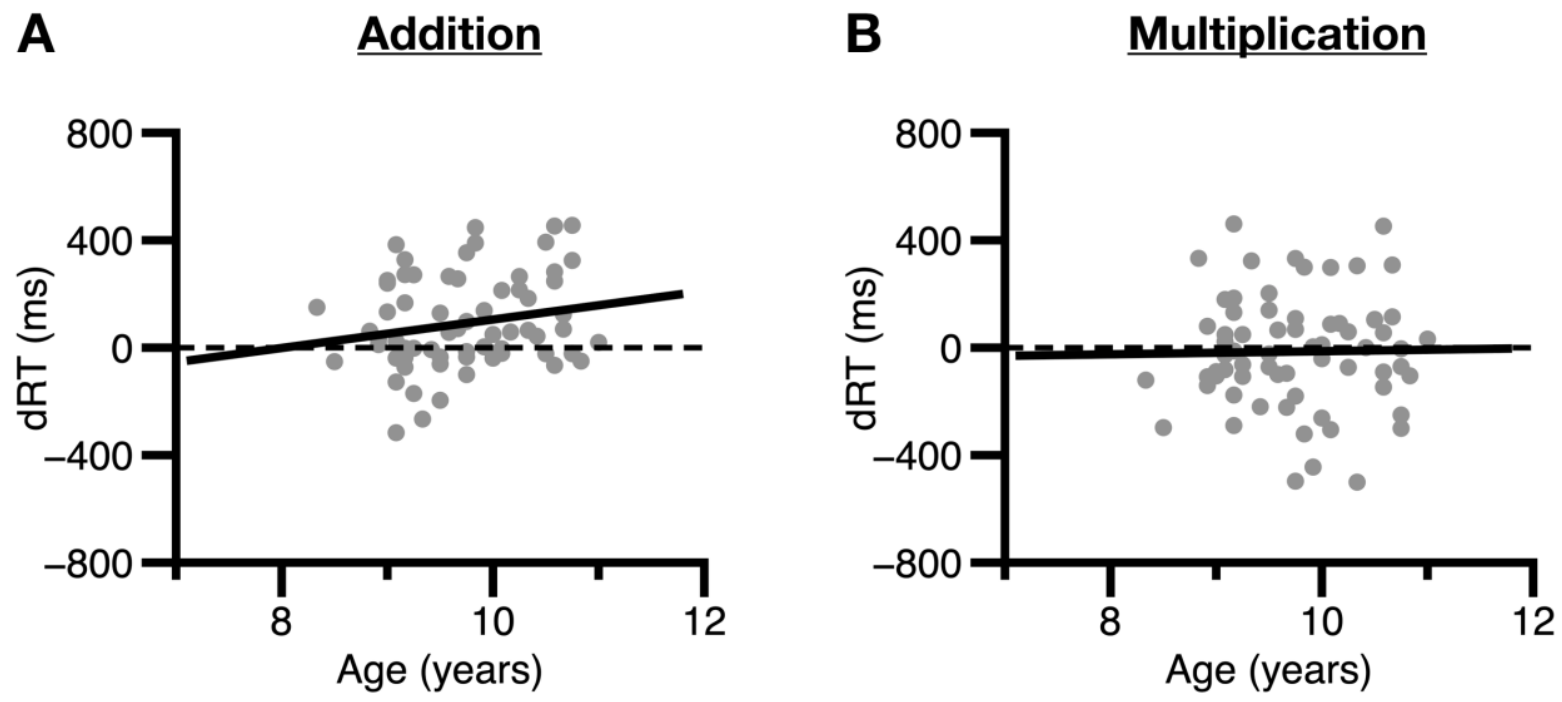

Figure 3. Relationship between age and dRTs for small arithmetic problems in Experiment 2. (A) Small addition problems. (B) Small multiplication problems. 


\section{Tables}

Table 1. Descriptive statistics across all children in Experiment 1.

\begin{tabular}{lcccc}
\hline Variable & Mean (SD) & Range min-max & Skewness & Kurtosis \\
\hline Age (years) & $9.10(.80)$ & $7.5-11$ & .52 & -.61 \\
WJ Math fluency & $43.61(12.69)$ & $23-82$ & .94 & .69 \\
dRT small addition problems & $-3.46(260.92)$ & $-651.87-621.14$ & .03 & .47 \\
dRT small subtraction problems & $25.49(241.25)$ & $-679.18-621.14$ & .25 & .09 \\
RT small addition problems & $1710.19(382.87)$ & $929.45-2661.13$ & .13 & -.40 \\
RT small subtraction problems & $1839.16(460.59)$ & $794.59-3195.25$ & .51 & .23 \\
\hline
\end{tabular}


Table 2. Regression analyses of dRTs for small addition and subtraction problems in Experiment 1.

\begin{tabular}{lccccc}
\hline & Estimate & SE Estimate & $\beta$ & $t$ & $p$ (two-tailed) \\
\hline$D V:$ Small addition problems & & & & & \\
Age & -.47 & 2.88 & -.02 & -.17 & .87 \\
WJ Math fluency & 1.29 & 2.87 & .06 & .45 & .65 \\
RT small addition problems & .08 & .09 & .11 & .83 & .41 \\
$F(3,97)=.25, p=.86, \mathrm{R}^{2}=.01$ & & & & & \\
& & & & & \\
$D V:$ Small Subtraction problems & & & & & .06 \\
Age & -4.94 & 2.62 & -.20 & -1.89 & .45 \\
WJ Math fluency & 1.84 & 2.43 & .10 & .76 & .92 \\
RT small subtraction problems & -.01 & .07 & -.01 & -.11 & \\
$F(3,97)=1.25, p=.29, \mathrm{R}^{2}=.04$ & & & & & \\
\hline
\end{tabular}

Note. $\mathrm{DV}=$ Dependent variable; $\mathrm{SE}=$ Standard Error. 
Table 3. Descriptive statistics across all children in Experiment 2.

\begin{tabular}{lcccc}
\hline Variable & Mean (SD) & Range min-max & Skewness & Kurtosis \\
\hline Age (years) & $9.74(.65)$ & $8.33-11$ & .10 & -.97 \\
WJ Math fluency (N=67) & $51.97(13.13)$ & $27-86$ & .72 & .06 \\
dRT Small addition problems & $91.47(173.94)$ & $-316.51-455.38$ & .29 & -.33 \\
dRT Small multiplication problems & $-14.61(209.89)$ & $-500.64-460.62$ & .02 & .06 \\
RT small addition problems & $1629.33(388.27)$ & $813.90-2600.42$ & .47 & .10 \\
RT small multiplication problems & $1727.22(355.62)$ & $900.99-2771.22$ & .30 & .56 \\
\hline
\end{tabular}


Table 4. Regression analyses of dRTs for small addition and multiplication problems in Experiment 2.

\begin{tabular}{|c|c|c|c|c|c|}
\hline & Estimate & SE Estimate & $\beta$ & $t$ & $p$ (two-tailed $)$ \\
\hline \multicolumn{6}{|l|}{$D V:$ Addition problems } \\
\hline Age & 4.54 & 2.72 & .20 & 1.67 & .099 \\
\hline Math fluency & .38 & 2.26 & .03 & .17 & .87 \\
\hline RT small addition problems & .07 & .08 & .15 & .90 & .37 \\
\hline \multicolumn{6}{|l|}{$F(3,63)=1.29, p=.29, \mathrm{R}^{2}=.06$} \\
\hline \multicolumn{6}{|l|}{ DV: Multiplication problems } \\
\hline Age & 1.02 & 3.51 & .04 & .29 & .77 \\
\hline Math fluency & -.92 & 2.62 & -.06 & -.35 & .73 \\
\hline RT small multiplication problems & .05 & .10 & .08 & -.47 & .64 \\
\hline$F(3,63)=.32, p=.79, \mathrm{R}^{2}=.02$ & & & & & \\
\hline
\end{tabular}

Note. $\mathrm{DV}=$ Dependent variable; $\mathrm{SE}=$ Standard Error. 